\title{
Ernst Ruska, prix Nobel de physique 1986
}

Médecine/sciences a déjà fait état de l'importance majeure du nouveau microscope à effet tunnel mis au point par G. Binnig et H. Rohrer. L'Académie de Stockholm a aussi, cette année, réparé un oubli en couronnant Ernst Ruska pour le microscope électronique dont le principe a été découvert il y a quelque cinquante ans.

En 1924, Louis de Broglie publiait une théorie selon laquelle une onde pouvait être associée à toute particule en mouvement. Cette théorie était la contrepartie de celle d'Einstein qui avait fait appel à l'aspect corpusculaire des ondes lumineuses pour expliquer l'effet photo-électrique. A la suite d'Einstein, honoré en 1921, Louis de Broglie recevait le prix Nobel en 1929, soit à peine cinq ans après la publication de ses travaux. Une des plus importantes applications de cette théorie ondulatoire fut le développement du microscope électronique dont les images fascinent l'imagination au même titre que celles des lointaines galaxies fournies par le téléscope. Ces images sont celles des interconnexions dans les circuits intégrés à très haute densité, celles des synapses du cerveau, celles de l'intérieur des mitochondries, ou encore celles du virus du SIDA.

Plus de cinquante ans après avoir réalisé la finesse du détail dans l'image du bord d'un diaphragme, Ernst Ruska vient à son tour d'être invité à Stockholm. Né en 1906, Ernst August Friedrich Ruska, diplômé des Hautes Écoles Techniques de Munich et de Berlin, travaillait à améliorer les caractéristiques du faisceau d'électrons d'un oscilloscope lorsqu'il imagina que ce même faisceau pouvait être la base d'ún nouvel $m / s n^{\circ} 2$ ool. 3, ftoriar 87 instrument dont la résolution dépasserait celle du microscope photonique par plusieurs ordres de grandeur. Il était sans doute à l'époque un des rares scientifiques à réaliser que les ondes de Louis de Broglie étaient autre chose qu'une formule mathématique. Son premier article soumis à Naturwissenschatten fut refusé. Pendant de longs mois, il lui a fallu se satisfaire au jour le jour d'un quantum additionnel de compréhension sur le cheminement des électrons à travers un champ magnétique, au milieu de collègues incrédules et de problèmes techniques quasi insurmontables auxquels il ne fallait pas penser, en particulier celui de réaliser des coupes ultra-fines et celui de fixer et de colorer adéquatement les tissus. Cependant grâce à ses efforts continus et en dépit du scepticisme de l'industrie, le microscope électronique est devenu, en 1939, un instrument de laboratoire disponible commercialement.

Son frère cadet Helmut, docteur en médecine, fut un ardent protagoniste de l'utilisation, en médecine et en biologie, de cet extraordinaire appareil. Aujourd'hui le microscope électronique est répandu à des milliers d'exemplaires à travers le monde. Étrangement, pour plusieurs microscopistes électroniciens, c'est l'attribution du prix Nobel qui leur fera découvrir Ernst Ruska.

Pour obtenir le prix Nobel il faut non seulement une brillante idée, un travail ardu, mais aussi parfois la longévité. Comme le disait ironiquement George Bernard Shaw, le Nobel est une bouée de sauvetage qu'on lance à un nageur parvenu sur le rivage.

\section{BRÈVES}

L'ANF, facteur atrial natriurétique, est sécrété en réponse à une expansion du volume circulatoire. Il active par un mécanisme inconnu une GMP-cyclase corpusculaire, entraînant une augmentation de la production de GMP cyclique à différents niveaux (glomérules rénaux, cortex surrénalien et cellules musculaires lisses des vaisseaux). Dans ces dernières cellules, l'effet de l'augmentation du GMPc est une relaxation. La vasopressine est, au contraire, une hormone hypertensive dont les récepteurs sont de deux types, $\mathrm{V} 1$, couplés à l'activation de l'adénylate cyclase, et V2, couplés à l'activation de la phospholipase C. La stimulation de V2 conduit aussi à l'inhibition de l'adénylate cyclase et de la guanylate cyclase, s'opposant donc à l'effet de l'ANF.

Nambi P, et al. Proc Natl Acad Sci USA 1986 ; 83 : 8492-5.

Le GMP cyclique est le second messager du facteur atrial natriurétique (ANF, atrial natriuretic factor). Telle est la conclusion fortement suggérée par les études de Huang C.L. et al qui montrent que l'ANF provoque une augmentation de la production glomérulaire de GMPc parallèle à l'hyperfiltration induite au niveau du glomérule par l'hormone et, qu'en outre, le GMPc et ses analogues injectés dans l'artère rénale reproduisent les effets physiologiques de l'ANF. Cette hormone étant la seule à augmenter l'excrétion urinaire de GMPc, la mesure de la concentration urinaire en GMPc pourrait permettre d'évaluer in vivo l'action glomérulaire de l'ANF. Huang CL, et al. Proc Natl Acad Sci USA $1986 ; 83$ : 8015-8.

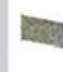

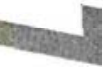

.

